\title{
No Lower Limit
}

National Cancer Institute

\section{Source}

National Cancer Institute. No Lower Limit. NCI Thesaurus. Code C155739.

A lower limit of the normal range does not exist. 